\title{
ASPECTOS QUE INFLUENCIAM A EXPRESSÃO DE COMPETÊNCIAS DE PERITOS CRIMINAIS
}

ASPECTS THAT INFLUENCE THE EXPRESSION OF COMPETENCIES OF CRIMINAL EXPERTS

ASPECTOS QUE INFLUENCIAN LA EXPRESIÓN DE COMPETENCIAS DE PERITOS CRIMINALES

\author{
LANA MONTEZANO \\ Doutoranda \\ Universidade de Brasília - Brasil \\ lanamontezano@gmail.com \\ ORCID: https://orcid.org/0000-0001-5288-4299 \\ GARDÊNIA DA SILVA ABBAD \\ Doutora \\ Universidade de Brasília - Brasil \\ gardeniaabbad@gmail.com \\ ORCID: https://orcid.org/0000-0003-0807-3549
}

Submetido em: 26/12/2017

Aprovado em: 14/02/2019

Doi: alcance.v26n1(Jan/Abr).p077-093

\section{RESUMO}

Este artigo tem por objetivo identificar aspectos que influenciam a expressão de competências de peritos criminais em uma organização pública brasileira. A pesquisa, exploratória-descritiva, com recorte transversal, foi feita com métodos qualitativos, por meio de pesquisa em documentos organizacionais relacionados ao tema e necessários à compreensão do contexto organizacional. Realizaram-se entrevistas com 19 gestores que atuam na área pericial e um grupo focal com técnicos da área de perícias criminais. Foram identificadas duas categorias de aspectos, as quais foram subdivididas em 11 subcategorias: Situacionais (suporte material, ambiente físico, políticas e práticas de gestão de pessoas, demanda de trabalho, apoio à gestão, suporte psicossocial, critérios de avaliação de resultados, estruturação organizacional e autonomia institucional) e individuais (motivação para o trabalho e estratégias de enfrentamento de situações adversas). Os participantes da pesquisa avaliaram mais favoravelmente 0 suporte material e 0 ambiente físico, e consideraram a motivação como uma importante variável preditiva da expressão de competências no trabalho. Os resultados mostraram que há aspectos de percepção de suporte organizacional semelhantes aos encontrados na literatura em outros contextos de trabalho e foram encontrados aspectos específicos não observados em estudos anteriores e não mensurados em contexto de atividade pericial.

Palavras-Chave: Competências. Perícia criminal. Suporte organizacional.

\section{ABSTRACT}

This article aims to identify aspects that influence the expression of competencies of professionals in the area of forensic science in a Brazilian public organization. This transversal, descriptive-exploratory study used qualitative methods, through research on organizational documents related to the theme that were necessary to understand the organizational context, interviews with nineteen managers who work in the forensic area, and a focus group with experts in the area of criminal forensics. Two categories of aspects (situational and individual) were identified, and these were further subdivided into eleven subcategories: Situational (material support, physical environment,

Revista Alcance - Eletrônica - vol. 26 - n. 1 - Jan./Abr. 2019 
policies and practices of people management, work demand, management support, psychosocial support, criteria for evaluation of the results, organizational structure and institutional autonomy) and individual (motivation for work and strategies for confronting adverse situations). The research participants evaluated support material and the physical environment more favorably, while also considering motivation as an important predictive variable to express the competencies of the job. The results showed that there are aspects of perception of organizational support similar to those found in the literature in other work contexts, and specific factors were found that were not observed in previous studies and not measured in the context of forensics activity.

Keywords: Competencies. Criminal forensics. Organizational support.

\section{RESUMEN}

Este artículo tiene por objetivo identificar aspectos que influencian en la expresión de competencias de peritos criminales en una organización pública brasileña. La investigación, exploratoria descriptiva, con recorte transversal, fue hecha con métodos cualitativos, por medio de investigación en documentos organizacionales relacionados al tema y necesarios a la comprensión del contexto organizacional. Se realizaron entrevistas con 19 gestores que actúan en el área pericial y un grupo focal con técnicos del área de pericias criminales. Fueron identificados dos categorías de aspectos, las cuales fueron subdivididas en 11 subcategorías: Situacionales (soporte material, ambiente físico, políticas y prácticas de gestión de personas, demanda de trabajo, apoyo a la gestión, soporte psicosocial, criterios de evaluación de resultados, estructuración organizacional y autonomía institucional) e individuales (motivación para el trabajo y estrategias de enfrentamiento en situaciones adversas). Los participantes de la investigación evaluaron más favorablemente el soporte material y el ambiente físico, y consideraron la motivación como una importante variable predictiva de la expresión de competencias en el trabajo. Los resultados mostraron que hay aspectos de percepción de soporte organizacional semejantes a los encontrados en la literatura en otros contextos de trabajo y fueron encontrados aspectos específicos no observados en estudios anteriores y no mensurados en contexto de actividad pericial.

Palabras clave: Competencias. Pericia criminal. Soporte organizacional.

\section{INTRODUÇÃO}

Atualmente, a importância da implementação da gestão de pessoas por competências tem sido adotada nas organizações devido ao fato de a sua finalidade viabilizar o aumento do desempenho organizacional e profissional, por meio da alocação e do desenvolvimento das competências profissionais alinhadas ao alcance dos objetivos organizacionais (BRANDÃO; GUIMARÃES; BORGES-ANDRADE, 2001; BRANDÃO, 2012). Isto se deve ao fato de que o bom desempenho do indivíduo (desempenho competente) é decorrente da expressão das competências do profissional do ambiente de trabalho (MONTEZANO; ABBAD; FREITAS, 2016).

Neste sentido, diversos estudos foram realizados com proposições de modelos de gestão por competências, tendo como principais etapas: (i) a definição das competências necessárias, (ii) a avaliação da existência destas competências nos profissionais, (iii) o desenvolvimento das lacunas existentes; e (iv) 0 acompanhamento do desempenho (CAMPION et al., 2011; UBEBA; SANTOS, 2008; ALVAREZ; TARRAGÓ, 2006; BRANDÃO; BAHRY, 2005). Apesar de alguns desses modelos contemplarem a necessidade da compreensão do estabelecimento prévio da estratégia organizacional que parte da premissa da compreensão do contexto organizacional, nenhum dos modelos relaciona aspectos das condições necessárias à expressão de competências dos indivíduos.

Face às lacunas identificadas, com base na literatura científica, tem-se a seguinte pergunta de pesquisa: quais são os aspectos que influenciam a expressão de competências bem-sucedidas de servidores públicos em um contexto específico de atuação profissional?

Dessa forma, este artigo tem como objetivo a identificação dos aspectos que influenciam a expressão de competências de peritos criminais no contexto de atuação específico de atividades ligadas às ciências forenses de uma organização pública. Além disso, foi possível identificar a percepção qualitativa de disponibilidade dos aspectos que influenciam a expressão de competências. 
Este estudo é parte do esforço de suprir as lacunas na produção de conhecimentos decorrentes da carência de estudos para: (1) investigar a relação de suporte organizacional com variáveis do comportamento organizacional (KURTESSIS et al., 2015), dentre elas as competências (BORGES-ANDRADE; PILATI, 2001); (2) identificar variáveis preditoras de competências individuais no contexto organizacional (MONTEZANO; COELHO JR; SILVA, 2015); (3) investigar quais características do contexto de trabalho, da equipe e da organização podem restringir ou facilitar a expressão de competências (BRANDÃO, 2007); (4) estudar quais fatores podem exercer influência sobre a expressão e a aquisição de competências no trabalho (BRANDÃO; BORGES-ANDRADE, 2007; COELHO JÚNIOR; MOURÃO; FAIAD; RÊGO, 2017); (5) investigar de que modo a percepção de suporte organizacional/variáveis contextuais afetam o desempenho (ABBAD O-C; PILATI; BORGES-ANDRADE, 1999; JIN; ZHONG, 2014), constructo correlato à expressão de competências no trabalho.

Além de suprir algumas lacunas na produção de conhecimento da área, este estudo permite uma reflexão sobre as práticas e os métodos de diagnóstico de competências adotadas pelas organizações, ao incorporar aspectos do contexto de trabalho que influenciam a expressão de competências. Além disto, os resultados da pesquisa podem estimular pesquisadores e profissionais a reverem as suas práticas, passando a observar e a analisar a influência de aspectos contextuais sobre comportamentos, desempenhos exibidos pelas pessoas nas organizações, de modo a estabeleceram as ações adequadas para proporcionar melhores resultados individuais e organizacionais.

Para tanto, o artigo apresenta a fundamentação teórica com a análise do constructo estudado, partindo de conceitos correlatos disponíveis na literatura e de suas variáveis antecedentes. Em seguida, o método descreve as características da pesquisa, a organização estudada, as técnicas de coleta e a análise de dados, com as respectivas amostras, além de reforçar a relevância do uso da triangulação de dados. Nas seções seguintes, encontram-se a descrição e a discussão dos resultados da pesquisa. Por fim, são apresentadas as contribuições e as limitações do estudo, bem como uma agenda de estudos futuros, visando suprir lacunas na produção de conhecimentos sobre os temas pesquisados.

\section{FUNDAMENTAÇÃO TEÓRICA}

Abbad, Freitas e Pilati (2006) sugeriram um modelo de investigação segundo o qual o desempenho competente (expressão de competências) é função de três tipos de condições no trabalho: (1) os ambientais (materiais, físicas e psicossociais), que dizem respeito ao "poder fazer"; (2) os saberes individuais (conhecimentos, habilidades e atitudes) relacionados ao "saber fazer"; e (3) as motivações (razões, metas, aspirações), ligadas ao "querer fazer". Estas condições podem ser associadas à teoria da atribuição da causa de Heider (1958), a qual estabelece que os comportamentos para gerar o desempenho são decorrentes de causas interna ou disposicionais, ou seja, é a vontade do próprio indivíduo em relação a alguma coisa; e de causas externas ou situacionais, ou seja, vinculadas ao ambiente em que o indivíduo está inserido.

Devido ao fato de a expressão de competências ser conceito correlato ao de desempenho no trabalho (MONTEZANO; ABBAD; FREITAS, 2016), pode-se considerar que as condições necessárias ao desempenho e à da expressão de competências sejam as mesmas.

Os estudos sobre a importância da influência do contexto no desempenho do indivíduo começaram na década de 80, sendo que Peters e O'Connor (1980) indicavam que as pessoas poderiam realizar suas tarefas com sucesso em decorrência de aspectos situacionais da organização. Mager e Pipe (1983) realizaram estudos sobre a compreensão de variáveis contextuais relacionados a possíveis problemas de desempenho (expressão de competências) e já ressaltavam a necessidade de um diagnóstico sistemático das causas destes problemas, podendo ser pela existência de possíveis obstáculos do contexto organizacional, tais como: modelo de gestão, perfil gerencial, falta de recursos materiais, patrimoniais e ferramentas, condições ambientais (temperatura, iluminação, ruídos, ergonomia, segurança e saúde), falta de informação e orientação para realização do trabalho, problemas de comunicação organizacional, ausência de feedback do desempenho, falta de autoridade, falta de tempo.

Brandão e Borges-Andrade (2007) afirmam que o suporte organizacional é uma variável contextual que influencia a expressão de competências dos profissionais que trabalham na organização. Brandão, BorgesAndrade e Guimarães (2012) acrescentam que a percepção dos funcionários acerca do suporte pode gerar tanto a manifestação das competências na melhoria do desempenho local de trabalho, quanto restringir ou anular esta relação, em caso de não ser percebida a oferta pela organização, ou mesmo por outras variáveis. Um exemplo

Revista Alcance - Eletrônica - vol. 26 - n. 1 - Jan./Abr. 2019 
desta lógica pode ser observado em estudos empíricos, como no caso em que a expressão de habilidades sociais depende de fatores contextuais de suporte organizacional (HOCHWARTER et al., 2006). Sendo assim, a percepção de suporte organizacional é um constructo correlato aos aspectos situacionais mais específicos, como aqueles que provavelmente influenciam a expressão de competências no contexto de atuação de periciais criminais.

Um dos percursores do estudo de suporte organizacional foi Eisenberger et al. (1986), que propuseram um instrumento para mensuração deste constructo, definido como a crença do indivíduo sobre o quanto a organização valoriza e retribui as suas contribuições e cuida do seu bem-estar no trabalho. Segundo esses autores, crenças favoráveis sobre suporte organizacional estão relacionadas positivamente com melhor desempenho e maior comprometimento do indivíduo com a organização. A escala original é unidimensional e possui 36 itens e, posteriormente, foi desenvolvida pelos autores uma escala reduzida de 17 itens.

Abbad O-C, Pilati e Borges-Andrade (1999) desenvolveram um instrumento brasileiro da percepção de suporte organizacional, baseado nos referenciais teóricos e definições de Eisenberger et al. (1986), com intuito de adaptar a medida à realidade das pesquisas nacionais. Nesse estudo, os autores identificaram que os profissionais têm a crença de que o suporte é visto como algo unidimensional e que a percepção brasileira dos aspectos que são considerados necessários como suporte é mais rica e detalhada. $O$ instrumento brasileiro é composto por 50 itens, os quais foram validados com altos índices de confiabilidade, e são relacionados a 4 fatores, sendo 13 itens de práticas de gestão de desempenho (percepções dos funcionários sobre definição de metas, reconhecimento de novas ideias e conhecimento das dificuldades em executar o trabalho eficazmente e esforço da organização para atualização dos profissionais em relação às novas tecnologias e aos processos de trabalho), 9 itens de carga de trabalho (percepções sobre carga de trabalho), 17 itens de suporte material (percepções sobre qualidade, disponibilidade e adequação dos recursos materiais e financeiros oferecidos pela organização) e 11 itens de práticas organizacionais de ascensão, promoção e salários (percepções as práticas de retribuição financeira, promoção e ascensão profissional). Queiroga, Brandão e Borges-Andrade (2015) propuseram uma escala unifatorial de percepção de suporte organizacional reduzida (EPSO-R) com 28 itens, com objetivo de diminuir 0 número de itens propostos por Abbad O-C, Pilati e Borges-Andrade (1999) e, com isto, obter maior índice de devolução de questionários respondidos.

Rhoadedes e Eisenberger (2002), em sua revisão de literatura sobre percepção de suporte organizacional, identificaram três principais categorias relacionadas ao suporte como variáveis antecedentes: justiça, apoio do supervisor, recompensas organizacionais (reconhecimento, pagamento e promoções) e condições de trabalho favoráveis (segurança no trabalho, autonomia, estressores da função, treinamento, tamanho da organização). Os autores identificaram que percepções favoráveis sobre suporte organizacional estão positivamente relacionadas ao comprometimento organizacional afetivo e ao desempenho, de modo que quanto melhores as percepções sobre o quanto a organização valoriza e retribui as contribuições do indivíduo para a organização, mais forte é o comprometimento afetivo e melhor é o desempenho do funcionário com a organização.

Alinhado a isso, Campos e Estivalete (2013) identificaram correlações altas entre a percepção de suporte organizacional e as bases do comprometimento organizacional, principalmente relacionadas aos aspectos afetivos e à obrigação em permanecer na organização; bem como correlação moderada entre a obrigação com 0 desempenho. Com isso, quanto maior for a percepção de suporte organizacional, maior será o comprometimento que contribuirá com o desempenho. Estudos recentes têm demonstrado que a percepção de suporte organizacional atua como variável moderadora positiva e significativa entre envolvimento (YONGXING et al., 2017) ou comprometimento (SEARS; ZHANG; HAN, 2016) com o desempenho do indivíduo.

Brandão e Borges-Andrade (2007) descreveram alguns exemplos de fatores que influenciam a expressão de competências, como apoio do gestor e colegas, incentivos e oportunidades de desempenho, e propriedades situacionais da organização. Brandão (2009) apresenta resultado de revisão de literatura em que identificou variáveis que podem influenciar o desempenho, tais como: políticas organizacionais de incentivos, recompensa e valorização; estrutura e tecnologia da organização; disponibilidade de orientações/informações direcionais; apoio psicossocial ao indivíduo; existência de suporte material; e qualidade do ambiente físico. Formiga e Souza (2014) indicam que o suporte organizacional está relacionado à promoção do desenvolvimento do profissional, disponibilização de recursos e autonomia para execução do trabalho, reconhecimento e visibilidade profissional. Lima e Silva (2015) identificaram fatores que influenciam o desenvolvimento das competências coletivas, que é uma premissa para que elas possam ser expressas no trabalho, sendo eles: dinâmica das atividades no contexto de trabalho; disponibilidade quantitativa de pessoal e do perfil necessário; características e disposições individuais; 
integração da equipe; comunicação; estrutura física; papel do gestor; e plano de carreira e política de desenvolvimento de pessoal.

Kurtessis et al. (2015), ao realizarem uma metanálise, constataram como variáveis antecedentes ao suporte organizacional aspectos relacionados ao contexto da organização, práticas de gestão de pessoas, condições de trabalho e liderança. E ainda identificaram que a orientação do profissional para a organização, 0 desempenho obtido e o bem-estar são variáveis consequentes ao suporte organizacional.

Pode-se perceber que os estudos, de um modo geral, relacionam o suporte organizacional a variáveis endógenas, ou seja, a aspectos internos da própria organização. No entanto, Ribeiro et al. (2017) extrapolam esta perspectiva ao considerarem variáveis exógenas relacionadas ao contexto organizacional influenciando na integração entre os recursos (ex: capital humano, cultura organizacional, relacionamento entre indivíduos, processos de trabalho) e as competências para a obtenção dos resultados de desempenho organizacional.

Abbad, Coelho Jr., Freitas e Pilati (2006) recomendaram o uso de múltiplas estratégias metodológicas para avaliar percepções de suporte organizacional, entre as quais questionários, entrevistas e pesquisas documentais, com vistas a identificar indicadores de suporte relacionados a práticas de valorização das contribuições dos profissionais, práticas de gestão de desempenho, cuidados com o bem-estar dos profissionais, coerência entre diretrizes, metas e ações organizacionais, espaço para manifestação de problemas pessoais, aspectos relacionados a ações de TD\&E, aspectos de oportunidade de ascensão profissional, reconhecimento e recompensas pelo esforço depreendido no trabalho.

Diante da importância de variáveis relativas à organização na predição de desempenho e comprometimento do indivíduo com a organização, esta pesquisa adotará como referencial a definição constitutiva do conceito de percepção de suporte organizacional, acrescida de dimensões ligadas a aspectos organizacionais específicos que influenciam a expressão de competências por profissionais de perícia. Neste trabalho, aspectos que influenciam a expressão de competências são definidos como as percepções de gestores e peritos sobre as condições organizacionais necessárias à expressão bem-sucedida de competências inerentes ao trabalho pericial. Contempla também as restrições situacionais que dificultam ou inviabilizam a expressão dessas competências no trabalho, além da percepção quanto a aspectos do próprio indivíduo, podendo afetar o desempenho e a expressão de competências periciais no trabalho. Sendo assim, a Figura 1 sintetiza este conceito dos aspectos que influenciam a expressão de competências, sendo que a identificação das variáveis situacionais e disposicionais foi o foco do estudo empírico desta pesquisa para o contexto específico de profissionais que atuam com ciências forenses.

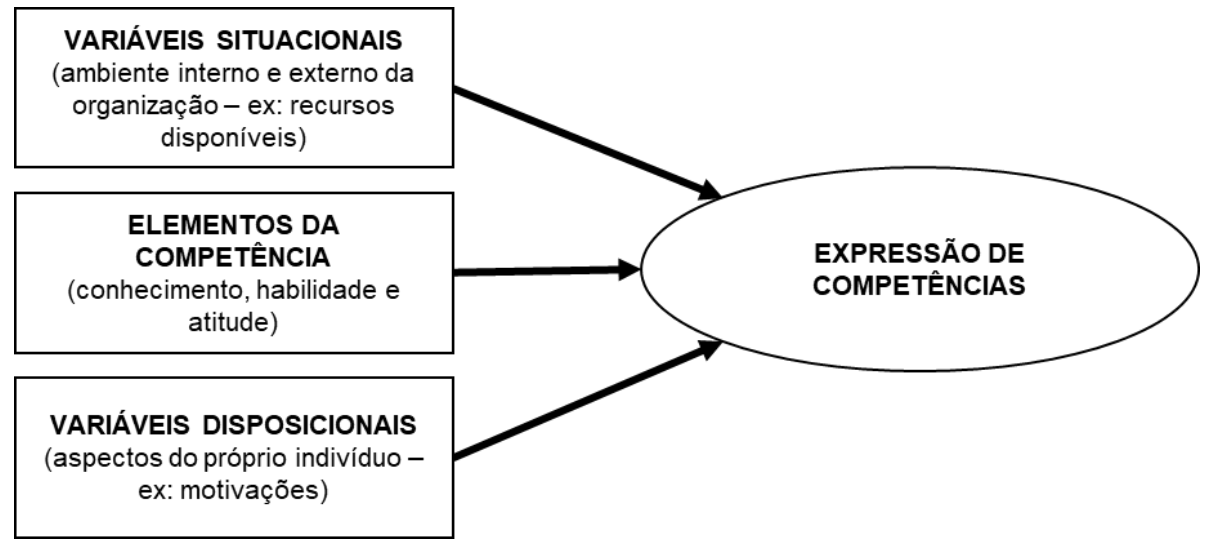

Figura 1. Condicionantes para expressão de competências Fonte: Elaboração própria

\section{MÉTODO}

Esta pesquisa caracteriza-se como teórica-empírica, de natureza exploratória-descritiva e com recorte temporal transversal. Foi utilizada abordagem qualitativa para identificação dos aspectos que influenciam a expressão de competências, por meio de três técnicas de coleta de dados (pesquisa documental, entrevistas e grupo focal), de modo a contemplar diferentes fontes documentais e humanas de informação, com percepções de

Revista Alcance - Eletrônica - vol. 26 - n. 1 - Jan./Abr. 2019 
gestores e técnicos, permitindo a triangulação dos dados, com vistas a buscar maior completude e verificar possíveis convergências de aspectos.

Trata-se de um estudo de caso realizado em uma organização pública, escolhida por conveniência, mediante autorização dos gestores responsáveis de $1^{\text {a }}$ nível até $03^{\mathrm{a}}$ nível de uma diretoria que atua em perícias baseadas em ciências forenses, de modo a permitir a análise em um contexto bem específico com particularidades vinculadas às suas atribuições. Essa diretoria tem como finalidade a busca da verdade dos fatos, por meio da produção de provas materiais com isenção e qualidade, utilizando-se de técnicas oriundas de ciências forenses. Além de executar as perícias, os profissionais também realizam pesquisas, desenvolvem novas técnicas para o aperfeiçoamento de procedimentos a serem utilizadas no exercício profissional, participam na formação de novos profissionais e elaboram normas para padronização e compartilhamento do conhecimento.

No momento da pesquisa, o quadro de pessoal da Diretoria estudada era de 345 servidores públicos, sendo 186 peritos, 74 em cargos administrativos e 82 de outros cargos do plano de carreira, os quais não serão especificados por questão de sigilo acordado com a organização. Sendo assim, alinhado ao objetivo da pesquisa, a população do estudo foi delimitada para os profissionais que atuam em perícias, dos quais 21 servidores possuem função gerencial nas áreas componentes da diretoria, sendo um de área normativa, dois em áreas de suporte, dois em áreas estratégicas e $16 \mathrm{em}$ áreas finalísticas, departamentalizadas por especialidades periciais. O perfil da população é caracterizado com $83,9 \%$ do sexo masculino e $100 \%$ com graduação, sendo que $60,2 \%$ possuem pós-graduação $(39,2 \%$ com mestrado, $9,1 \%$ com mestrado e doutorado, $8,6 \%$ com especialização e $3,2 \%$ com doutorado), o que confere a eles uma característica de profissionais com elevado nível de formação educacional.

A amostragem utilizada para participar das coletas de dados foi não probabilística por conveniência, em função da disponibilidade de acesso aos gestores das unidades componentes da diretoria estudada para as entrevistas e aos peritos que aceitaram participar dos grupos focais. Em ambas as coletas de dados primários obteve-se a concordância de todos os sujeitos para gravar em áudio as sessões de entrevista e grupos focais e transcrever esse material para posterior análise de conteúdo. Os pesquisadores responsáveis por este estudo garantiram sigilo das informações individuais e se comprometeram em não revelar o nome da organização estudada. A amostra utilizada é detalhada especificamente em cada técnica de coleta de dados, descrita a seguir.

Na pesquisa documental, foram consultados documentos normativos internos, manuais, planejamento estratégico e relatório de atividades. Além disso, foram analisadas quatro dissertações de mestrado profissional desenvolvidas por servidores da referida Diretoria que tratavam de temas relacionados a esta pesquisa. A leitura dos documentos foi realizada mediante comparação com as definições do referencial teórico para identificar aspectos com indicativo de influência na expressão de competências, os quais foram registrados como temas em um quadro para serem agrupados em categorias temáticas, seguindo as diretrizes de Bardin (2011). Além disso, permitiu compreender o contexto organizacional do locus de pesquisa, por meio da caracterização das atividades e das atribuições da diretoria que atuam com as perícias.

As percepções dos gestores e dos peritos foram coletadas por meio de entrevistas individuais semiestruturadas e grupos focais, respectivamente. Foram realizadas 19 entrevistas individuais com gestores das áreas finalísticas (periciais), normativa e estratégicas. A entrevista foi conduzida presencialmente, com o apoio de um roteiro semiestruturado, composto por quatro questões abertas que tratavam da percepção dos entrevistados sobre os aspectos que podem influenciar a expressão de competências no trabalho, tanto sob o ponto de vista do gestor quanto do técnico que atua em atividades baseadas nas ciências forenses, sendo elas: quais as condições necessárias para os peritos expressarem com sucesso suas competências profissionais? Quais as condições necessárias para que um gestor consiga expressar com sucesso as competências profissionais que possui? Estas condições existem na sua área de trabalho? Quais melhorias poderiam ser adotadas pela área para facilitar a expressão de competências? O perfil dos gestores entrevistados caracteriza-se como 89,5\% do sexo masculino; com idade variando de 37 a 55 anos, sendo a maioria (42,1\%) entre 35 e 39 anos; que tomaram posse no cargo finalístico há 13,5 anos, em média, sendo a maioria (53,6\%) entre 10 e 14 anos; com experiência média em cargo de gestão de 5,2 anos. Dos gestores entrevistados, $89,5 \%$ possuem pós-graduação, inclusive dentre eles $57,9 \%$ de mestres, $15,8 \%$ de doutores e $15,8 \%$ de especialistas.

Foi realizada uma variação da técnica de grupo focal com 13 servidores que atuam diretamente nas atividades finalísticas de perícias apoiadas em ciências forenses para coletar a percepção segundo a ótica desses profissionais, com uso de roteiro estruturado com duas perguntas que foram definidas, a partir da análise preliminar das informações das entrevistas (Quais os aspectos da organização contribuem para sua expressão de 
competências e quais os aspectos do próprio indivíduo contribuem para que possam expressar as competências). O grupo focal foi realizado em três etapas: (1) respostas individuais às duas questões do roteiro; (2) discussão em subgrupos de 4 pessoas sobre as respostas das questões; e (3) discussão em plenária com todos os participantes para consolidação e busca de pontos de convergência e divergências nas respostas do grupo às mesmas questões. Esta estratégia de coleta favoreceu a discussão final em função da riqueza das percepções que emergiram nas etapas anteriores e facilitou a busca de consenso do grupo. 0 perfil dos participantes do grupo focal foi de $77 \%$ do sexo masculino, com média de 41 anos de idade, que tomaram posse no cargo há 10,3 anos, em medida, e 92,3\% com pós-graduação, sendo a maioria com mestrado (53,8\%).

Os dados coletados foram transcritos gerando um corpus total de 32.009 palavras em 43 páginas, sendo 31.752 palavras oriundas das entrevistas e 257 do grupo focal, em que foi gravada e transcrita somente a última etapa da discussão plenária. Em seguida, os dados foram submetidos à análise categorial temática de Bardin (2011), considerando critérios de homogeneidade, exclusão e pertinência, inicialmente com quatro juízes de um Grupo de Pesquisa de uma Universidade Federal que estuda temáticas de Treinamento, Desenvolvimento e Educação (TD\&E). Após a análise por juízes, os dados foram analisados e consolidados. $O$ resultado da análise gerou os temas apontados como aspectos, sendo que estes foram agrupados em categorias de conteúdo temáticas por núcleo de sentido similares. A categorização levou em consideração a teoria de assuntos correlatos.

A partir do material consolidado na análise de conteúdo, foi realizada entrevista coletiva com 10 servidores da organização, tendo representantes tanto de participantes da entrevista quanto do grupo focal, com intuito de verificar a adequação e a completude das informações coletadas junto ao público-alvo da pesquisa, como forma de validação dos aspectos. Na oportunidade, validaram a compreensão dos termos utilizados para nomenclatura das categorias e das respectivas definições constitutivas, sendo realizados ajustes que se adequassem ao contexto da realidade da Organização.

\section{RESULTADOS}

A partir da análise dos dados coletados, foi possível definir duas categorias de aspectos que influenciam a expressão de competências, resultado que se alinha à literatura especializada (Heider, 1958; Brandão \& BorgesAndrade, 2007) quanto à necessidade de oferta de condições de suporte organizacional (situacionais) e em relação ao próprio indivíduo (individuais), a partir da análise dos relatos apresentados pelos participantes na pesquisa. No entanto, identificou-se a necessidade de estabelecer subcategorias relacionadas a cada uma delas, para dar maior riqueza e clareza dos dados obtidos, sendo definidas nove subcategorias dos aspectos situacionais e duas de disposicionais. A Tabela 1 apresenta os nomes e as definições constitutivas dos aspectos que influenciam a expressão de competências.

\section{Tabela 1.}

Categorias e subcategorias dos aspectos que influenciam a expressão de competências

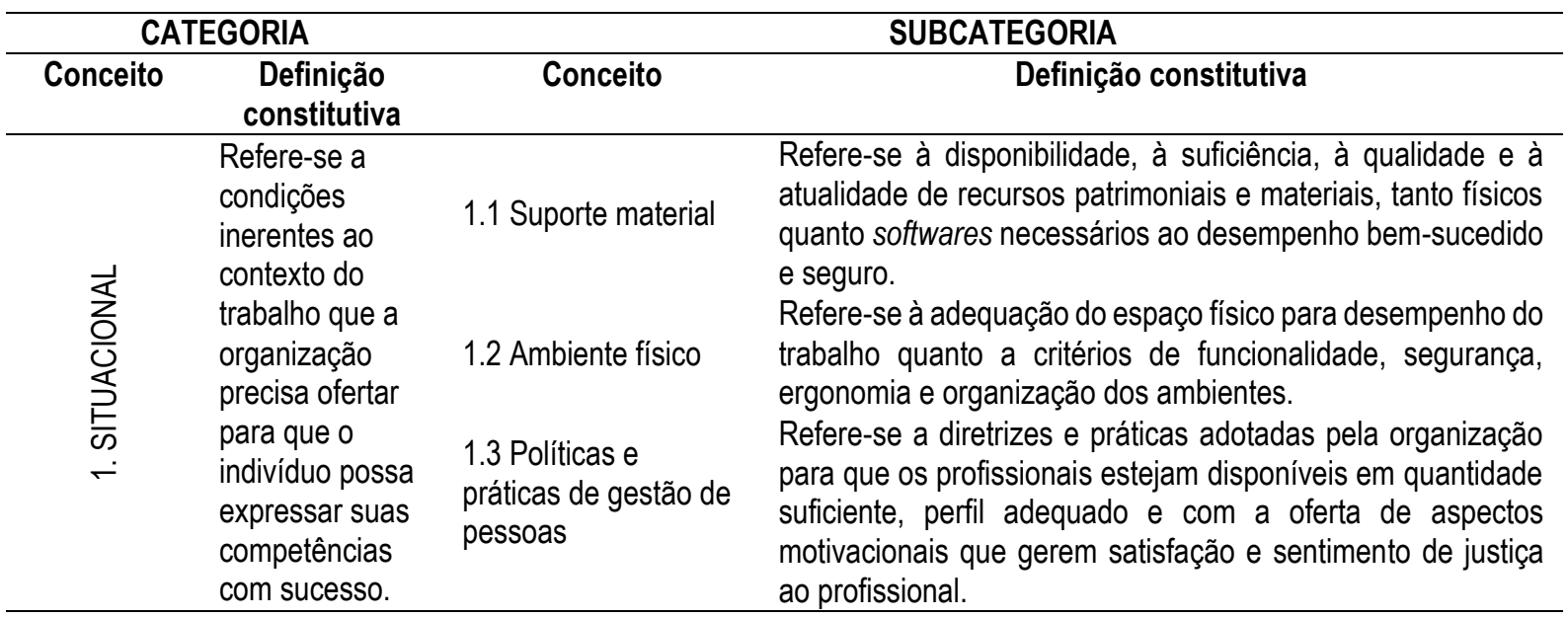

(Continua) 
(Conclusão)

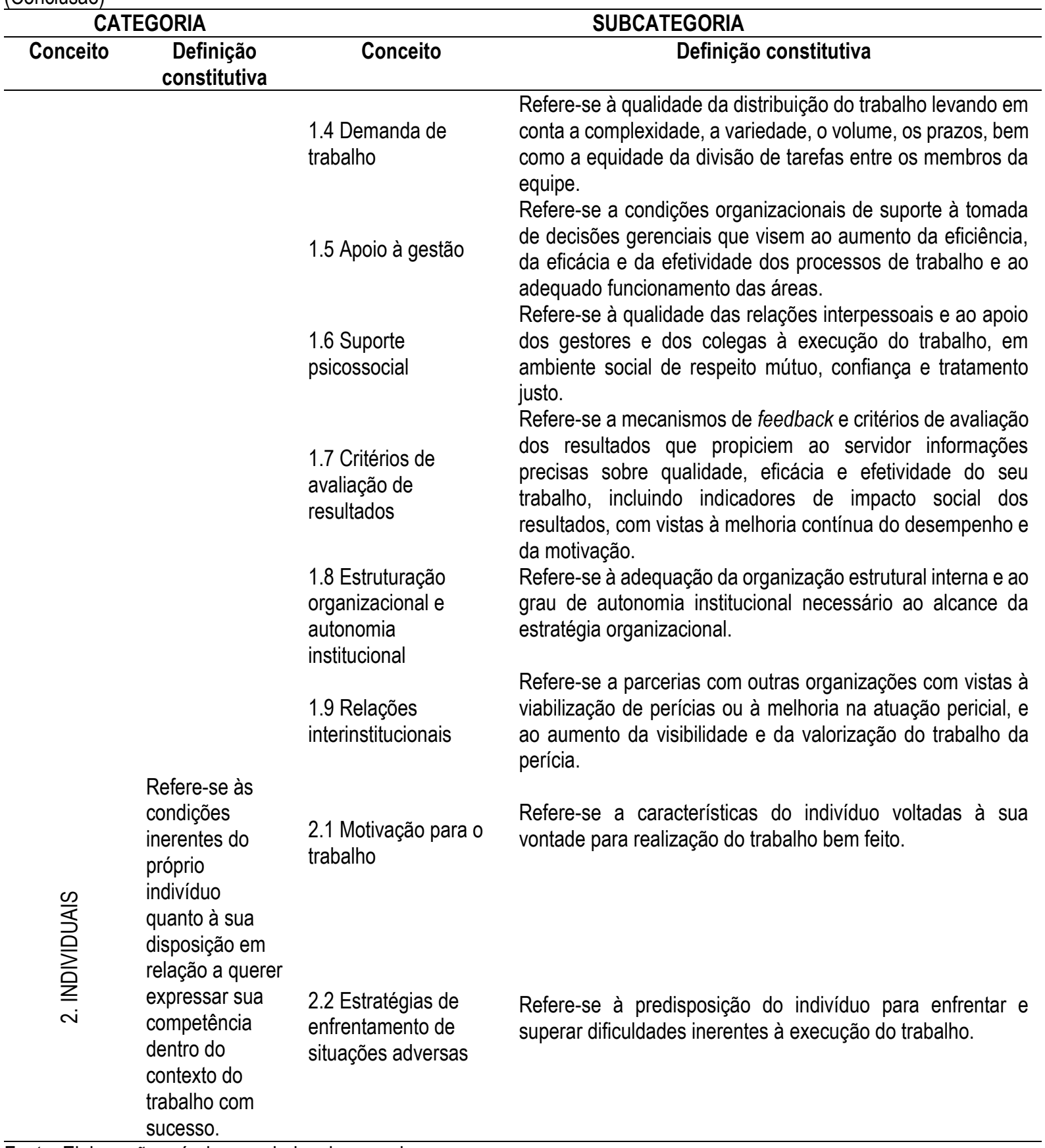

Fonte: Elaboração própria com dados da pesquisa.

A Tabela 2 exemplifica os relatos que subsidiaram a subcategorização, incluindo a identificação do sujeito com uso do código "GFT" para grupo focal com técnicos e "EG" para entrevista com gestor e numeração aleatória ao gestor. Ressalva-se que algumas exemplificações foram limitadas, devido ao fato de que a inserção dos relatos poderia comprometer o sigilo da identificação da organização, por isso não foram incluídos. 


\section{Tabela 2.}

Exemplos de relatos para definição das subcategorias dos aspectos que influenciam a expressão de competências

\begin{tabular}{|c|c|}
\hline Subcategoria & Exemplos de relatos \\
\hline Suporte Material & $\begin{array}{l}\text { - "A pessoa tem que ter disponível o equipamento no momento adequado... com a manutenção } \\
\text { adequada, com os insumos necessários" (EG5). } \\
\text { - "Precisa de uma mesa... um computador com duas telas" (EG7). } \\
\text { - "Ter o computador também adequado, em alguns casos isso é fundamental... essa infraestrutura } \\
\text { tem que ser dada, sem ela o desempenho fica comprometido" (EG10). }\end{array}$ \\
\hline Ambiente Físico & $\begin{array}{l}\text { - "Você ter uma infraestrutura física, eu diria que isso é importante." (EG6). } \\
\text { - "Tem a segurança do prédio" (EG11). } \\
\text { - "A qualidade do ambiente de trabalho é fundamental para que uma pessoa tenha condição } \\
\text { de desenvolver ao máximo ali as suas habilidades, as suas competências" (EG12). } \\
\text { - "A gente fala de ergonomia e tal... faz diferença. Se eu tiver bem acomodado no microscópio } \\
\text { faz diferença, dá para trabalhar uma hora no microscópio, mas se eu tiver em pé, incômodo } \\
\text { assim não consigo trabalhar mais de } 20 \text { minutos, tenho que descansar" (EG13). }\end{array}$ \\
\hline $\begin{array}{l}\text { Políticas e práticas de } \\
\text { gestão de pessoas }\end{array}$ & $\begin{array}{l}\text { - "Fazer um trabalho que seja reconhecido" (EG1). } \\
\text { - "Da capacitação, que é um fator necessário, a pessoa tem que estar preparada" (EG2). } \\
\text { - "Mas existe a preocupação de que se tenha uma análise do serviço psiquiátrico e psicológico } \\
\text { aqui" (EG8). } \\
\text { - "A questão da valorização dos profissionais" (EG12). }\end{array}$ \\
\hline Demanda de trabalho & $\begin{array}{l}\text { - "O volume de trabalho muito grande ele impede a gente de fazer, é assim restritivo para expandir } \\
\text { ou expressar essa competência" (EG3). }\end{array}$ \\
\hline Apoio à gestão & $\begin{array}{l}\text { - "Então a gente procura uniformizar os modelos" (EG2). } \\
\text { - "Ter mais condição de você definir onde vai pôr o recurso" (EG4). } \\
\text { - "A normatização e a normalização são peças chave para isso acontecer" (EG7). }\end{array}$ \\
\hline Suporte psicossocial & $\begin{array}{l}\text { - "Acho que relação interpessoal mesmo" (EG5). } \\
\text { - "Você precisa desta equipe integrada" (EG6). } \\
\text { - "Você tem que ter o suporte das chefias" (EG10). }\end{array}$ \\
\hline $\begin{array}{l}\text { Critérios de avaliação } \\
\text { de resultados }\end{array}$ & $\begin{array}{l}\text { - "Perceber a efetividade daquilo que você está fazendo" (EG12). } \\
\text { - "Então acho que ter esse feedback" (EG12). } \\
\text { - "Consegue avaliar se os resultados estão bons" (EG10). } \\
\text { - "Impacta na produtividade" (EG4). } \\
\text { - "Feedback da qualidade dos laudos" (GFT). }\end{array}$ \\
\hline $\begin{array}{l}\text { Estruturação } \\
\text { organizacional e } \\
\text { autonomia } \\
\text { institucional }\end{array}$ & - "Se o nosso organograma, a nossa estrutura gerencial é ideal" (EG5). \\
\hline $\begin{array}{l}\text { Relações } \\
\text { interinstitucionais }\end{array}$ & $\begin{array}{l}\text { - "Autonomia, mas mais a nível organizacional" (EG4). } \\
\text { - "Depender de apoio de outras instituições, de apoio de outros locais" (EG12). }\end{array}$ \\
\hline $\begin{array}{l}\text { Motivação para o } \\
\text { trabalho }\end{array}$ & $\begin{array}{l}\text { - "Demonstrar a importância do trabalho deles e fazer com que eles realmente produzam" (EG2). } \\
\text { - "Eu vejo mais motivação como uma coisa importante" (EG3). } \\
\text { - "A principal seria essa motivação" (EG6). }\end{array}$ \\
\hline $\begin{array}{l}\text { Estratégias de } \\
\text { enfrentamento de } \\
\text { situações adversas }\end{array}$ & $\begin{array}{l}\text { - "Mas é uma situação assim, que hoje a gente depende muito mais da capacidade individual de } \\
\text { cada um de se desligar, de se abstrair, dessa questão" (EG8). } \\
\text { - "É uma carga emocional muito grande" (EG17). }\end{array}$ \\
\hline
\end{tabular}

Fonte: Elaboração própria com dados da pesquisa.

Além da categorização, os relatos possibilitaram a identificação dos principais aspectos (facetas) relacionados a cada uma das subcategorias, os quais foram quantificados por tipo de fonte de coleta de dados. A

Revista Alcance - Eletrônica - vol. 26 - n. 1 - Jan./Abr. 2019 
Tabela 3 apresenta a quantificação, em ordem decrescente de recorrência por subcategoria, bem como a frequência da recorrência de relatos relacionados a cada subcategoria nas entrevistas dos gestores.

Tabela 3.

Frequência de aspectos identificados em cada subcategoria por fonte de coleta de dados

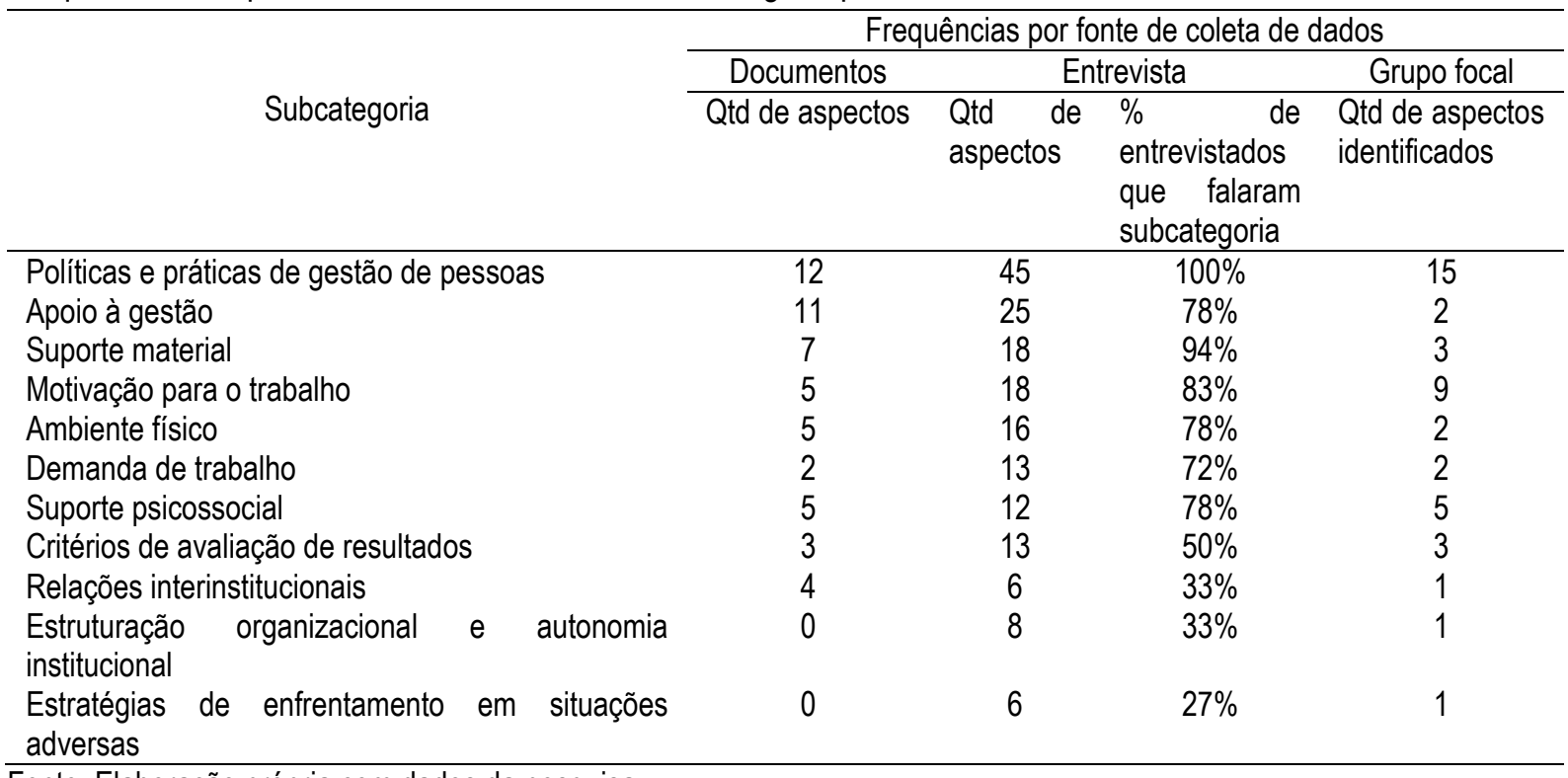

Fonte: Elaboração própria com dados da pesquisa.

Uma das subcategorias ligadas a características individuais (Estratégias de enfrentamento em situações adversas) não foi frequente nas falas dos entrevistados, indicando que os aspectos mais influentes parecem ser os de suporte organizacional (aspectos situacionais). Já a motivação para o trabalho ficou em $4^{0}$ lugar de quantitativo de aspectos identificados, o que remete a um ponto relevante na percepção dos participantes na influência da expressão de competências. Há indícios de que motivação e capacidade de enfrentamento de situações adversas influenciam a expressão de competências no trabalho do perito. No entanto, não foram identificados estudos empíricos na literatura que associam as variáveis individuais com a expressão de competências. Em função disto, recomendam-se mais estudos sobre esses aspectos pessoais e uma extensa análise da literatura que possibilite a distinção entre diversos aspectos relacionados à motivação "no" e "para" o trabalho, bem como a formulação de uma definição teoricamente válida desses conceitos. São, pois, necessários mais estudos teóricos e empíricos que confiram maior validade a esses achados.

Pode-se perceber que 0 uso de diferentes fontes de coletas contribuiu para identificação de maior variedade de aspectos, além de permitir a identificação de subcategorias que poderiam não ter sido obtidas, caso fosse utilizado somente a fonte documental de coleta. Outro ponto relevante quanto à metodologia dos achados é o fato de que as entrevistas possibilitaram identificar uma maior diversidade de aspectos do que o grupo focal. Além de permitir maior variação e abrangência de informações, a triangulação de informações obtidas pelas três fontes (documentos, gestores e peritos) também possibilitou a identificação de pontos de convergência entre as percepções de técnicos e gestores e nos documentos. A Tabela 4 contém exemplos dos aspectos convergentes. 


\section{Tabela 4.}

Exemplos de aspectos que influenciam expressão de competências por subcategorias

\begin{tabular}{|c|c|}
\hline Subcategoria & Exemplos de aspectos \\
\hline Suporte Material & $\begin{array}{l}\text { Disponibilidade de bons equipamentos, materiais e insumos } \\
\text { Disponibilidade de recursos tecnológicos necessários } \\
\text { Programas computacionais específicos das áreas }\end{array}$ \\
\hline Ambiente Físico & $\begin{array}{l}\text { Adequação do tipo de espaço físico para realização do trabalho } \\
\text { Segurança no trabalho (incluindo de armazenagem segura de materiais de laboratório, } \\
\text { acústica para realização de exames) } \\
\text { Infraestrutura física adequada }\end{array}$ \\
\hline $\begin{array}{l}\text { Políticas e práticas de } \\
\text { gestão de pessoas }\end{array}$ & $\begin{array}{l}\text { Capacitação formal continuada e específica para atuação pericial } \\
\text { Reconhecimento e valorização do profissional } \\
\text { Meritocracia }\end{array}$ \\
\hline Demanda de trabalho & $\begin{array}{l}\text { Equidade na divisão do trabalho } \\
\text { Justiça na distribuição do trabalho } \\
\text { Carga de trabalho adequada ao tipo/complexidade de demanda }\end{array}$ \\
\hline Apoio à gestão & $\begin{array}{l}\text { Normatização } \\
\text { Padronização do trabalho (ex: modelos) } \\
\text { Gestão de processos de trabalho }\end{array}$ \\
\hline Suporte psicossocial & $\begin{array}{l}\text { Apoio da chefia } \\
\text { Autonomia na realização do trabalho } \\
\text { Trabalho colaborativo }\end{array}$ \\
\hline $\begin{array}{l}\text { Critérios de avaliação de } \\
\text { resultados }\end{array}$ & $\begin{array}{l}\text { Qualidade no trabalho } \\
\text { Resultado/efetividade dos laudos } \\
\text { Associação de qualidade, produtividade e efetividade } \\
\text { Feedback do trabalho }\end{array}$ \\
\hline $\begin{array}{l}\text { Estruturação } \\
\text { organizacional e } \\
\text { autonomia institucional }\end{array}$ & $\begin{array}{l}\text { Clareza das responsabilidades das áreas } \\
\text { Divisão de responsabilidades adequadas à capacidade de gestão }\end{array}$ \\
\hline Relações interinstitucionais & Parcerias institucionais (Cooperação técnica e fomento à pesquisa) \\
\hline Motivação para o trabalho & $\begin{array}{l}\text { Disposição para realização do trabalho } \\
\text { Preocupação com o impacto do seu trabalho } \\
\text { Preocupação com a importância do trabalho desenvolvido } \\
\text { Querer fazer }\end{array}$ \\
\hline $\begin{array}{l}\text { Estratégias de } \\
\text { enfrentamento de } \\
\text { situações adversas }\end{array}$ & $\begin{array}{l}\text { Disposição para enfrentar problemas/desafios } \\
\text { Resiliência } \\
\text { Superação de sofrimento no trabalho } \\
\text { Controle emocional }\end{array}$ \\
\hline
\end{tabular}

Fonte: Elaboração própria com dados da pesquisa.

Conforme consta na Tabela 3, a subcategoria de políticas e práticas de gestão de pessoas foi a que surgiu maior diversidade de temas, além de ser a que teve mais evocações. Alguns aspectos foram considerados positivos, como o investimento da organização em capacitações, mas é um dos aspectos que consideram maior necessidade de aperfeiçoamento para que possa contribuir de forma mais efetiva com a expressão de competências dos profissionais na geração de resultados bens sucedidos, conforme indicado em fala de gestor: 
"Isso aí é uma questão que nós precisamos melhorar, eu acho que essa nossa questão de política de gestão de pessoas dentro da... não só dentro do nosso órgão, mas dentro dos órgãos públicos é falho" (EG1).

Ao analisar os relatos das perspectivas dos entrevistados, foi possível identificar que, de um modo geral, os aspectos de suporte material foram considerados como as melhores condições ofertadas pela organização, se comparados com outras instituições nacionais e internacionais que atuam com o mesmo tipo de trabalho ou até com outros órgãos públicos, conforme afirmado pelos gestores exemplificado na seguinte fala:

"O (nome da unidade) é um oásis... assim da parte técnica, o equipamento, os sistemas você não vai encontrar nada melhor no Brasil... (exemplifica comparações com outros países)... então nessa questão de equipamentos, de sistemas é o que tem de melhor"(EG16).

Além de identificar os aspectos do ambiente físico, pode-se perceber que a avaliação dos gestores quanto à sua oferta pela organização está relacionada ao tempo de existência da área, sendo que as mais novas ainda estão se estruturando para ter salas específicas para realização de determinados exames, por exemplo, ou devido a expansão do atendimento de novas demandas que necessitam de um ambiente propício para ser estruturado. Isto pode ser observado nas seguintes evocações dos gestores:

"Nós temos uma ótima estrutura e não tem do que se queixar... eu acho que o principal seria mais esta questão laboratorial (a unidade não possui laboratório). Isto, já está sendo previsto."

"Eu acho que esta parte de infraestrutura está tranquilo, a gente tá bem servido" (EG6).

No caso dos aspectos individuais, a subcategoria de motivação foi ressaltada como fundamental para que os servidores possam expressar as competências, relacionando a vontade de fazer o trabalho a gostar do que se faz, de dar importância ao seu trabalho. Algumas verbalizações das entrevistas podem ilustrar este enfoque:

\footnotetext{
"A principal seria essa motivação" (EG6).

"Mas quando tu estás motivado... a gente tirava leite de pedra...por isso que eu digo assim que esse é o primeiro, porque daí você constrói o resto" (EG6).
}

Já a subcategoria "estratégias de enfrentamento de situações adversas" foi criada pela especificidade de determinadas atuações dos profissionais em demandas que exigem um autocontrole e lidar com o trabalho sem adoecer.

\section{DISCUSSÃO}

O estudo confirmou a importância de suporte organizacional e de aspectos situacionais como antecedentes da expressão de competências, tanto das condições ofertadas pela própria organização, que foram denominados de situacionais (MAGER; PIPE, 1983; ABBAD O-C et al., 1999; ABBAD et al., 2006; BRANDÃO; BORGES-ANDRADE, 2007; FORMIGA; SOUZA, 2014; QUEIROGA; BRANDÃO; BORGES-ANDRADE, 2015), quanto aos que são inerentes do próprio indivíduo em querer fazer algo (ABBAD et al., 2006; BRANDÃO; BORGES-ANDRADE, 2007; LIMA; SILVA, 2015). Pode-se verificar alinhamento entre os achados e a literatura quanto aos aspectos situacionais, conforme indicado na Tabela 5. 


\section{Tabela 5.}

Comparação de aspectos que influenciam a expressão de competências: resultados da pesquisa $\mathrm{X}$ literatura

\begin{tabular}{|c|c|c|}
\hline $\begin{array}{l}\text { Aspectos identificados na } \\
\text { pesquisa }\end{array}$ & $\begin{array}{l}\text { Aspectos identificados em outras } \\
\text { pesquisas }\end{array}$ & Pesquisas \\
\hline \multirow{5}{*}{ Suporte material } & \multirow{5}{*}{$\begin{array}{l}\text { Necessidade de recursos materiais, } \\
\text { patrimoniais e ferramentas }\end{array}$} & Mager e Pipe (1983) \\
\hline & & Abbad O-C et al. (1999) \\
\hline & & Abbad et al. (2006) \\
\hline & & Brandão e Borges Andrade (2007) \\
\hline & & Queiroga, Brandão e Borges-Andrade (2015) \\
\hline \multirow{8}{*}{ Ambiente físico } & \multirow{8}{*}{ Condições ambientais } & Mager e Pipe (1983) \\
\hline & & Abbad O-C et al. (1999) \\
\hline & & Rhoadedes e Eisenberger (2002) \\
\hline & & Abbad et al. (2006) \\
\hline & & Brandão e Borges Andrade (2007) \\
\hline & & Formiga e Souza (2014) \\
\hline & & Lima e Silva (2015) \\
\hline & & Queiroga, Brandão e Borges-Andrade (2015) \\
\hline \multirow{10}{*}{$\begin{array}{l}\text { Políticas e práticas de gestão } \\
\text { de pessoas }\end{array}$} & \multirow{4}{*}{ Preparação para o trabalho } & Rhoadedes e Eisenberger (2002) \\
\hline & & Abbad et al. (2006) \\
\hline & & Lima e Silva (2015) \\
\hline & & Queiroga, Brandão e Borges-Andrade (2015) \\
\hline & \multirow{6}{*}{$\begin{array}{l}\text { Políticas organizacionais de } \\
\text { incentivos, recompensa e valorização }\end{array}$} & Abbad O-C et al. (1999) \\
\hline & & Rhoadedes e Eisenberger (2002) \\
\hline & & Brandão e Borges Andrade (2007) \\
\hline & & Formiga e Souza (2014) \\
\hline & & Lima e Silva (2015) \\
\hline & & Queiroga, Brandão e Borges-Andrade (2015) \\
\hline \multirow{4}{*}{ Demanda de trabalho } & \multirow{4}{*}{ Disponibilidade/falta de tempo } & Mager e Pipe (1983) \\
\hline & & Abbad O-C et al. (1999) \\
\hline & & Abbad et al. (2006) \\
\hline & & Queiroga, Brandão e Borges-Andrade (2015) \\
\hline \multirow{6}{*}{ Apoio à gestão } & \multirow{2}{*}{ Modelo de gestão } & Mager e Pipe (1983) \\
\hline & & Queiroga, Brandão e Borges-Andrade (2015) \\
\hline & \multirow{4}{*}{$\begin{array}{l}\text { Falta de informação para realização } \\
\text { do trabalho }\end{array}$} & Mager e Pipe (1983) \\
\hline & & Abbad et al. (2006) \\
\hline & & Queiroga, Brandão e Borges-Andrade (2015) \\
\hline & & Brandão e Borges Andrade (2007) \\
\hline \multirow{5}{*}{ Suporte psicossocial } & \multirow{5}{*}{ Apoio psicossocial } & Abbad O-C et al. (1999) \\
\hline & & Rhoadedes e Eisenberger (2002) \\
\hline & & Brandão e Borges Andrade (2007) \\
\hline & & Lima e Silva (2015) \\
\hline & & Queiroga, Brandão e Borges-Andrade (2015) \\
\hline \multirow{3}{*}{$\begin{array}{l}\text { Critérios de avaliação de } \\
\text { resultados }\end{array}$} & \multirow{3}{*}{$\begin{array}{l}\text { Necessidade de feedback/foco em } \\
\text { resultado }\end{array}$} & Mager e Pipe (1983) \\
\hline & & Abbad O-C et al. (1999) \\
\hline & & Queiroga, Brandão e Borges-Andrade (2015) \\
\hline
\end{tabular}


(Conclusão)

\begin{tabular}{lll}
\hline $\begin{array}{l}\text { Estruturação organizacional e } \\
\text { autonomia institucional }\end{array}$ & $\begin{array}{l}\text { Estrutura e tecnologia de } \\
\text { organização }\end{array}$ & $\begin{array}{l}\text { Rhoadedes e Eisenberger (2002) } \\
\text { Brandão e Borges Andrade (2007) }\end{array}$ \\
$\begin{array}{ll}\text { Serviços requeridos e ajuda dos } \\
\text { outros } \\
\text { Velações interinstitucionais exógenas ao contexto } \\
\text { organizacional }\end{array}$ & Abbad et al. (2006) \\
\hline
\end{tabular}

Fonte: Elaborada pelas autoras.

Esta comparação permitiu identificar na literatura aspectos relacionados a todas as categorias de aspectos situacionais, apesar de serem em diferentes pesquisas. Isso reforça o fato da dependência do contexto do trabalho para a definição de quais serão os aspectos que irão influenciar a expressão de competências, o que remete à necessidade de um instrumento específico com itens que mensurem todos os aspectos identificados para o caso de peritos criminais, em vez de usar um instrumento previamente validado pela literatura. No entanto, não foram identificados aspectos com variáveis individuais relacionando com a expressão de competências. Não foram identificadas na literatura a influência de variáveis motivacionais e a de estratégias de enfrentamento com a expressão de competências. Por isso, não serão discutidas em profundidade as relações entre esses dois tipos de aspectos individuais e expressão de competências.

O estudo contribuiu em identificar nove categorias de aspectos situacionais, tendo maior quantidade do que as existentes na literatura, como no estudo de Abbad O-C et al. (1999), que havia identificado quatro fatores. Esses aspectos são ligados a aspectos inerentes ao contexto do trabalho pericial, não contemplados nos instrumentos de suporte organizacional, porque estes não visam avaliar percepções específicas como as que são o foco deste estudo. No entanto, observa-se que existem variáveis deste estudo em 6 subcategorias dos achados desta pesquisa, pois devido ao contexto do trabalho e à percepção dos participantes que validaram as categorias, foi considerado deixar em aspectos distintos. Isso remete à necessidade de maior investigação quanto à melhor categorização dos aspectos organizacionais que influenciam a expressão de competências, podendo ser feito por meio de análise fatorial com dados coletados, a partir de um instrumento desenvolvido com os resultados desta pesquisa.

Ao analisar os dados coletados durante a pesquisa, pode-se verificar que, assim como apresentado por Abbad et al. (2006), foram identificados dados que remetem às três dimensões das restrições situacionais: indisponibilidade (ex: disponibilidade de energia elétrica, insumos no momento do exame), quantidade (ex: EPI suficiente) e qualidade (computadores adequados).

Ainda que não fosse o objetivo inicial do estudo, os resultados obtidos com uso de diferentes técnicas indicam a pertinência desta escolha metodológica, pois permitiu a triangulação das informações, tanto como forma de confirmação das diferentes perspectivas como complemento de dados necessários ao resultado obtido. A pesquisa documental por si só não produziria informações suficientes para a definição dos aspectos, uma vez que não foi possivel identificar dados relacionados a duas subcategorias (Estruturação organizacional e autonomia institucional da categoria situacional e Estratégias de enfrentamento em situações adversas da categoria individual). Mesmo assim, o uso desta técnica agregou valor no sentido de permitir conhecer o contexto da organização, bem como identificar temas que não haviam sido observados nas outras duas técnicas de coleta em relação às quatro subcategorias (Motivação, Relações institucionais, Suporte psicossocial e Apoio à gestão).

\section{CONCLUSÕES}

Foi possivel identificar aspectos que influenciam a expressão de competências profissionais no contexto de trabalho de uma organização pública brasileira, contribuindo para suprir lacunas da literatura quanto à necessidade de investigar a existência de variáveis antecedentes situacionais e disposicionais que influenciam a expressão de competências (Brandão \& Borges-Andrade, 2007; Montezano et al., 2015), inclusive com categorias mais abrangentes do que as existentes na literatura (Abbad O-C et al., 1999; Queiroga, Brandão \& BorgesAndrade, 2015), e com a identificação de variáveis exógenas à organização que podem influenciar na expressão das competências (relacionamento interinstitucional), conforme apontado por Ribeiro et al. (2017). E, ainda, foi 
possível contribuir com a literatura com a proposição de definição constitutiva para os aspectos identificados como tendo influência sobre a expressão de competências.

Isso remete a uma reflexão quanto à necessidade de repensar o conceito de diagnóstico de competências, de modo a incorporar nos modelos de diagnóstico abordados na literatura etapas que contemplem a identificação de fatores que influenciam a expressão de competências, e assim permitir a proposição de soluções mais adequadas às efetivas causas das lacunas (competências ou dos aspectos).

O estudo contribui para constatar que as variáveis que influenciam a expressão de competências profissionais são multiníveis, ou seja, no nível micro, dependem de aspectos próprios do indivíduo (ex: motivação), no meso, de aspectos da equipe (ex: apoio dos colegas, de processos de trabalhos) e no nível macro, da própria estrutura organizacional e dos aspectos da gestão. E ainda vai além do contexto da própria organização, necessitando de aspectos do ambiente externo, como o relacionamento interinstitucional. Com isso, novas investigações deverão considerar variáveis multiníveis que influenciam a expressão de competências dos indivíduos.

Esta pesquisa também trouxe contribuições metodológicas para a produção do conhecimento, no que diz respeito à aplicação da técnica do grupo focal em um formato adaptado que gerou resultado mais completo do grupo final, após passar pelas etapas de reflexão individual e discussões em subgrupos. Esta técnica é pouco utilizada em estudos relacionados à temática de competências, conforme apontado na revisão da literatura feita por Montezano et al. (2015).

O uso de múltiplas técnicas de coleta, oriundas de diferentes fontes de dados secundários (documentos) e primários (percepção de gestores nas entrevistas e técnicos nos grupos focais), também foi uma contribuição por adotar a triangulação, conforme recomendações metodológicas da literatura, o que contribuiu para ter mais consistência e confiabilidade nos dados gerados e analisados, além de permitir identificar alinhamento de percepções entre técnicos, gestores e organização pelos seus documentos formais.

Como limitação da pesquisa, tem-se a não identificação de produções que tenham investigado aspectos individuais associados à expressão de competências para permitir a comparação dos achados da pesquisa com as publicações científicas. Por outro lado, este aspecto pode ser uma oportunidade de suprir a lacuna de estudos que permitam identificar se estes aspectos são variáveis antecedentes da expressão de competências. Para tanto, faz-se necessário pesquisar a existência de estudos que investiguem constructos correlatos aos aspectos individuais com o desempenho humano.

Para continuidade desta pesquisa, com intuito de validar as categorias e as subcategorias, tem-se a necessidade de construir um instrumento de diagnóstico dos aspectos que influenciam a expressão de competências profissionais, para verificar se as definições operacionais de mensuração das subcategorias como aspectos que influenciam a expressão de competências possuem validade empírica, bem como para avaliar 0 quanto os profissionais percebem sua ocorrência na organização (referentes a aspectos situacionais) ou neles próprios (individuais). Para construção de subescalas específicas para o contexto pericial, deverão ser ampliadas as análises da produção científica sobre os temas pesquisados, bem como realizadas pesquisas empíricas que evidenciem a validade da medida por meio das seguintes estratégias (i) busca de validade incremental por meio da comparação com outros instrumentos correlatos; (ii) validação por juízes, realizada com especialistas na temática e em construção de instrumento; (iii) validação semântica com os profissionais da organização para adequação ao seu contexto de trabalho, bem como verificar a compreensão das instruções do instrumento e dos itens, e a adequação do uso da escala de avaliação a ser utilizada; (iv) análise fatorial exploratória e confirmatória, inclusive com análise fatorial multigrupo, para verificar se os fatores são aplicáveis a diferentes áreas e localidades de atuação no Brasil; (v) validade externa, por meio da aplicação do instrumento ajustado nas etapas anteriores em outras organizações públicas que também atuam com demandas periciais, sendo utilizados os mesmos testes estatísticos de análises fatoriais.

$E$, ainda, é recomendável investigar a existência de correlações entre a ocorrência destes aspectos que influenciam a expressão de competências com as lacunas de competências profissionais. Com base nisto, será possivel propor soluções para o resultado do diagnóstico, direcionando esforços ao que realmente for necessário (ações de aprendizagem ou outras ações para aperfeiçoar os aspectos - variáveis antecedentes). E, finalmente, é recomendável acompanhar o quanto a implementação dessas soluções resulta na evolução da percepção dos indivíduos sobre restrições situacionais e individuais à expressão de competências no trabalho. 


\section{REFERÊNCIAS}

Abbad, G. S., Coelho Jr., F. A., Freitas, I. A., \& Pilati, R. (2006). Medidas de suporte em avaliação de TD\&E. In Borges-Andrade, J. E., Abbad, G. S., \& Mourão, L. (Org.) Treinamento, Desenvolvimento e Educação em Organizações e Trabalho: fundamentos para a gestão de pessoas. (pp. 395-421). Porto Alegre: Artmed.

Abbad, G. S., Freitas, I. A., \& Pilati, R. (2006). Contexto de trabalho, desempenho competente e necessidades em TD\&E. In Borges-Andrade, J. E., Abbad, G. S., \& Mourão, L. (Org.) Treinamento, Desenvolvimento e Educação em Organizações e Trabalho: fundamentos para a gestão de pessoas. (pp. 231-254). Porto Alegre: Artmed.

Abbad O-C, G., Pilati, R., Borges-Andrade, J. E. (1999) Percepção de suporte organizacional: desenvolvimento e validação de um questionário. Revista de Administração Contemporânea, 3(2), 29-51.

Alvarez, Y. Y. D., \& Tarragó, N. S. (2006). Identificación de competencias en edición para los profesionales de la información. ACIMED, 14(2), 1-19.

Bardin, L. (2011). Análise de conteúdo: edição revista e ampliada. São Paulo: Edições 70.

Borges-Andrade, J. E., \& Pilati, R. (2001). Comprometimento atitudinal e comportamental: relações com suporte e imagens nas organizações. Revista de Administração Contemporânea, 5(3), 85-106.

Brandão, H. P. (2007). Competências no trabalho: uma análise da produção científica brasileira. Estudo de Psicologia, 12(2), 149-158.

Brandão, H. P. (2009). Aprendizagem, Contexto, Competência e Desempenho: um estudo multinível (Tese de doutorado). Universidade de Brasília, Brasília, DF. Brasil.

Brandão, H. P. (2012). Mapeamento de Competências: métodos, técnicas e aplicações em gestão de pessoas. São Paulo: Atlas.

Brandão, H. P., \& Bahry, C. P. (2005). Gestão por competências: métodos e técnicas para mapeamento de competências. Revista do Serviço Público, 2(56), 179-194.

Brandão, H. P., \& Borges-Andrade, J. E. (2007). Causas e efeitos da expressão de competências no trabalho: para entender melhor a noção de competência. Revista de Administração Mackenzie, 8(3), 32-49.

Brandão, H. P., Borges-Andrade, J. E., \& Guimarães, T. A. (2012). Desempenho organizacional e suas relações com competências gerenciais, suporte organizacional e treinamento. Revista de Administração de São Paulo, 47 (4), 523-539.

Brandão, H. P., Guimarães, T. de A., \& Borges-Andrade, J. E. (2001). Competências profissionais relevantes à qualidade no atendimento bancário. Revista de Administração Pública, 356(6). 61-31.

Campion, M. A., Fink, A. A., Ruggeberg, B. J., Carr, L., Phillips, G. M., \& Odman, R. B. (2011). Doing competencies well: best practices in competency modeling. Personnel Psychology, 64, 225-262.

Campos, S. A. P., \& Estivalete, V. F. B. (2013). O trabalho de quem ensina: suporte social, suporte organizacional e comprometimento. Revista Alcance, 20(1), 58-78.

Coelho Júnior, F. A., Mourão, L., Faiad, C., \& Rêgo, M. C. B. (2017). Escala sobre Competências de tutores na Educação a Distância: Evidências de Validade Interna e de Constructo. Encontro da ANPAD, São Paulo/SP.

Eisenberger, E., Huntington, R., Hutchison, S.M Sowa, D. (1986). Perceived Organizational Support. Journal of Applied Psychology, 71(3), 500-507.

Formiga, N. S., Souza, M. A. (2014). Comprovação empírica de uma medida psicológica sobre a percepção do suporte organizacional em trabalhadores de diferentes empresas. Bol. Acad. Paulista de Psicologia, 34(87). 510522.

Heider, F. (1958). The psychology of interpersonal relations. New York: John Wiley \& Sons.

Hochwarter, W. A., Witt, L. A., Treadway, D. C., \& Ferris, G. R. (2006). The interaction of social skill and organizational support on job performance. Journal of applied psychology, 91(2), 482-489.

Jin, L., \& Zhong, Y. (2014). Contextual factors affecting the influence of perceived organizational support on team innovative performance. Social behavior and personality, 42(3), 517-528. 
Kurtessis, J. N., Eisenberger, R., Ford, M. T., Buffardi, L. C., Stewart, K. A., \& Adis, C. A. (2015). Perceived Organizational Support: A Meta-Analytic Evaluation of Organizational Support Theory. Journal of Management, 131.

Lima, J. O., \& Silva, A. B. (2015). Determinantes do desenvolvimento de competências coletivas na gestão de pessoas. Revista de Administração Mackenzie, 16(5), 41-67.

Mager, R., \& Pipe, P. (1983). Análise de problemas de desempenho ou "você precisa realmente querer". Porto Alegre: Globo.

Montezano, L., Abbad, G. S., Freitas, P. F. P. (2016). Modelagem de competências profissionais de organização pública que atua no ramo de ciências forenses. XL Encontro da ANPAD, Costa do Sauípe/BA.

Montezano, L., Silva, D. L. B, \& Coelho Júnior, F. A. (2015). Competências humanas no trabalho: a evolução das publicações nacionais no novo milênio. XXXIX Encontro da ANPAD, Belo Horizonte/MG.

Peters, L. H., \& O'Connors, E. J. (1980). Situational constraints and work outcomes: the influence of frequently overlooked constructo. Academy of Management Review, 5(3), 391-397.

Queiroga, F., Brandão, H. P, e Borges-Andrade, J. E. (2015). Escala de percepção de suporte organizacional versão reduzida. In: Puente-Palacios, K. \& Peixoto, A. L. A. (Org.) Ferramentas de diagnóstico para organizações e trabalho: um olhar a partir da psicologia. Porto Alegre: Artmed. pp. 272-283.

Rhoades, L., \& Eisenberger, R. (2002). Perceived Organizational Support: a review of the literature. Journal of Applied Psychology, 87(4), 698-714.

Ribeiro, J. S. A. N, Soares, M. A. C., Jurza, P. H., Ziviani, F., \& Neves, J. T. R. (2017). Gestão do conhecimento e desempenho organizacional: integração dinâmica entre competências e recursos. Perspectivas em Gestão \& Conhecimento, 7(Número Especial), 4-17.

Sears, G. J., Zhang, H., \& Han, Y. (2016). Negative affectivity as a moderator of perceived organizational support - work outcome relationships. Personality and individual diferences, 98, 257-260.

Ubeda, C. L., \& Santos, F. C. A. (2008). Os principais desafios da gestão de competências humanas em um instituto público de pesquisa. Revista Gestão e Produção, 12(1), 189-199.

Yongxing, G., Hongfei, D., Baoguo, X., \& Lei, M. (2017). Work engagement and job performance: the moderating role of perceived organizational support. Anales de psicología, 33(3), 708-713. 